\title{
Association between the Cardio-Ankle Vascular Index and Diabetes Mellitus-Related Peripheral Arterial Disease in Chronic Hemodialysis Patients
}

\author{
Fumihiko Furuya $^{a} \quad$ Ai Motosugi $^{b}$ Kazutaka Haraguchib Shunichiro Hanai ${ }^{a}$ \\ Toshihisa Ishii $^{\text {a }}$ Yasuno Yamaguchi $^{\text {a }}$ Kenichiro Kitamura ${ }^{a}$ \\ ${ }^{a}$ Third Department of Internal Medicine, Interdisciplinary Graduate School of Medicine and Engineering, University \\ of Yamanashi, Chuo, Yamanashi, Japan; ${ }^{b}$ Haraguchi Clinic, Kai, Yamanashi, Japan
}

\section{Keywords}

Diabetes · Hemodialysis · Peripheral arterial disease

\section{Abstract}

Background: Peripheral arterial disease (PAD) has increased in association with the increase in the numbers of patients with kidney disease or diabetes. The aim of this study was to assess the prevalence of PAD in hemodialysis patients with diabetes. Methods: To examine the usefulness of the cardioankle vascular index (CAVI) to screen for the presence of PAD, cross-sectional studies of 100 patients undergoing chronic hemodialysis were performed. The CAVI and other inflammatory markers were evaluated. Results: The CAVI was markedly elevated in patients with a history of PAD or cardiovascular disease. When dialysis patients were classified on the basis of CAVI quartiles, increased CAVI was associated with other risk factors for PAD. Conclusion: The prevalence of PAD is high in elderly diabetic patients on hemodialysis. The present findings suggest that the CAVI can be a useful index that predicts the occurrence of macrovascular complications in dialysis patients with diabetes.

(c) 2019 The Author(s)

Published by S. Karger AG, Basel

\section{KARGER}

E-Mail karger@karger.com www.karger.com/bpu

\section{Introduction}

Peripheral arterial disease (PAD) is a major cause of acute and chronic illness [1]. Patients with PAD are at high risk of developing cardiovascular events, such as myocardial infarction, stroke, or cardiovascular death. For example, it has been shown in nearly 10,000 patients with vascular disease, diabetes mellitus, or age over 65 years plus at least 2 additional cardiovascular risk factors that, after a follow-up period of 6 months, approximately 1 in 8 patients with PAD had a vascular event or required cardiovascular hospitalization, a rate that was considerably higher than in patients without PAD [2]. A recent report also indicated that patients with PAD exhibited a fourfold increase in overall mortality and eightfold increased risk of cardiovascular death [3]. Thus, the presence of PAD is associated with a reduced quality of life and disability.

It should be noted that a significant proportion of patients with PAD are asymptomatic. More than $20 \%$ of individuals with PAD in the general population and $10 \%$ of patients over 65 years of age diagnosed with PAD by general practitioners did not show classical symptoms and 
were diagnosed by ankle-branchial index (ABI) measurement alone [4].

Patients with chronic kidney disease (CKD) are at increased risk for the development and progression of vascular calcification and atherosclerosis [5]. The common and noninvasive methods to diagnose arterial stiffness in CKD patients currently include brachial-ankle pulse wave velocity (baPWV; via arterial tonometry or Doppler ultrasound) [6] and the cardio-ankle vascular index (CAVI). Measurement of baPWV is a useful method to diagnose arterial stiffness in any part of body; however, its major limitation is that it is affected by changes in blood pressure during measurement. The CAVI, which represents the stiffness of the aorta and the femoral and tibial arteries, is essentially independent of blood pressure [7]. Several recent studies indicated that there is a significant correlation between the CAVI and baPWV [8]. In addition, it has been reported that the CAVI is a clinical marker of arterial stiffness in patients with essential hypertension, diabetes, obesity, and metabolic syndrome [9].

In the present study, the relationships between risk factors for PAD and atherosclerotic progression, evaluated by the CAVI, a new index of arterial stiffness, were investigated in hemodialysis patients. Since dialysis patients are at increased risk for arterial stiffness, the CAVI could be used as a screening test to detect the risk of PAD.

\section{Subjects and Methods}

\section{Subjects}

The subjects of this study were 100 outpatients undergoing chronic hemodialysis. The ABI or CAVI was measured using CAVI-Vasera VS-1500 (Fukuda Denshi, Tokyo, Japan). The mean value of the right and left ABIs was used for analysis, and values of 1.0 or more were considered normal for the ABI. The CAVI and ABI were measured during hemodialysis at 30 to $60 \mathrm{~min}$ into the session [7]. Skin pressure perfusion (SPP) measurements of the soles of the feet were taken $1 \mathrm{~h}$ after the hemodialysis session. SPP was measured with a laser Doppler probe enclosed within the bladder and a cuff wrapped around the foot sole using a SensiLase PAD3000 (Kaneka Medix Corp., Osaka, Japan). The patients were positioned in a supine position at room temperature [10].

Data are expressed as means $\pm \mathrm{SD}$. Statistical analyses were carried out using ANOVA, and $p<0.05$ was considered significant.

The study protocol was approved by the Ethics Committees of the University of Yamanashi and Haraguchi Clinic in accordance with the Declaration of Helsinki. Written informed consent was obtained from each patient.

\section{Principle of the CAVI and Method of Measurement}

The CAVI is based on the stiffness parameter $(\beta)$, which expresses blood pressure-independent vascular stiffness and is calculated as follows [11]: $\beta=(\ln [\mathrm{Ps} / \mathrm{Pd}])(\mathrm{D} / \Delta \mathrm{D})$, where $\ln$ indicates
Table 1. Clinical characteristics of study population with or without diabetes

\begin{tabular}{llll}
\hline & $\begin{array}{l}\text { Diabetes } \\
(n=52)\end{array}$ & $\begin{array}{l}\text { Non-diabetes } \\
(n=48)\end{array}$ & \\
\hline Age, years, means \pm SD & $59 \pm 14$ & $62 \pm 15$ & ns \\
Male, $n(\%)$ & $37(71.5)$ & $32(66.7)$ & ns \\
Obesity, $n(\%)$ & $14(26.9)$ & $2(4.2)$ & $0.01^{*}$ \\
Smoke, $n(\%)$ & $34(65.4)$ & $28(58.3)$ & ns \\
Hypertension, $n(\%)$ & $44(84.6)$ & $46(95.8)$ & ns \\
Dyslipidemia, $n(\%)$ & $20(38.5)$ & $16(33.3)$ & ns \\
Hyperuricemia, $n(\%)$ & $27(51.9)$ & $35(72.9)$ & ns \\
\hline
\end{tabular}

$* p<0.05$.

Obesity: BMI $>25 \mathrm{~kg} / \mathrm{m}^{2}$. Dyslipidemia: LDL-cholesterol $>40$ $\mathrm{mg} / \mathrm{dL}$ or HDL-cholesterol $<40 \mathrm{mg} / \mathrm{dL}$. Hyperuricemia: uric acid $>7.5 \mathrm{mg} / \mathrm{dL}$. Smoke: including smoking history

ns, no significant.

natural log; Ps, systolic pressure; Pd, diastolic pressure; D, diameter; and $\Delta \mathrm{D}$, change of diameter.

The relationship between volume elastic modulus and PWV is expressed by Bramwell-Hill's formula [12] as the following equation: $\mathrm{Z}^{2}=(\Delta \mathrm{P} / \rho)(\mathrm{V} / \Delta \mathrm{V})$, where $\mathrm{Z}$ indicates cardio-ankle $\mathrm{PWV}$ $(\mathrm{m} / \mathrm{s}) ; \Delta \mathrm{P}$, pulse pressure; $\mathrm{V}$, volume of blood vessel; $\Delta \mathrm{V}$, change of $\mathrm{V}$; and $\rho$, blood density $\left(1.03 \times 0^{3} \mathrm{~kg} / \mathrm{m}^{3}\right)$.

Because a cylindrical model using the length and diameter can be applied for blood vessels, the following equation is given: $\mathrm{V} /$ $\Delta \mathrm{V}=\mathrm{D} / 2 \Delta \mathrm{D}$. Then, the above 2 equations give the following equations: $\mathrm{D} / \Delta \mathrm{D}=2 \rho \mathrm{Z}^{2} / \Delta \mathrm{P}, \beta=(2 \rho / \Delta \mathrm{P})(\ln [\mathrm{Ps} / \mathrm{Pd}]) \mathrm{Z}^{2}$. Thus, the stiffness parameter $(\beta)$ can be easily calculated by measures of blood pressure and cardio-ankle PWV. The index, named the CAVI, is expressed as $\alpha \beta+b$ to convert $\beta$ to a value that approximates conventional aortic PWV for each patient. While these constants ( $\mathrm{a}$ and $\mathrm{b}$ ), proposed by the company (Fukuda Denshi), have still not been made public, the CAVI is easily measured using the CAVI Vasera VS-1000.

\section{Results}

A total of 100 patients gave their informed consent to participate in the study. Patients were divided into 2 groups, with or without diabetes. Fifty-two of the 100 patients had diabetes mellitus. Obesity was more prevalent in the patients with diabetes. Table 1 summarizes the background characteristics of the 100 patients of this study. There were no differences in the presence of hypertension, dyslipidemia, or hyperuricemia. History of smoking was comparable between the groups. As shown in Figure 1, the CAVI was significantly higher in diabetic patients $(10.02 \pm 0.8$ vs. $9.62 \pm 0.8)$.

Simple regression analysis indicated that the CAVI or $\mathrm{ABI}$ was correlated with the age of the patients on hemo- 


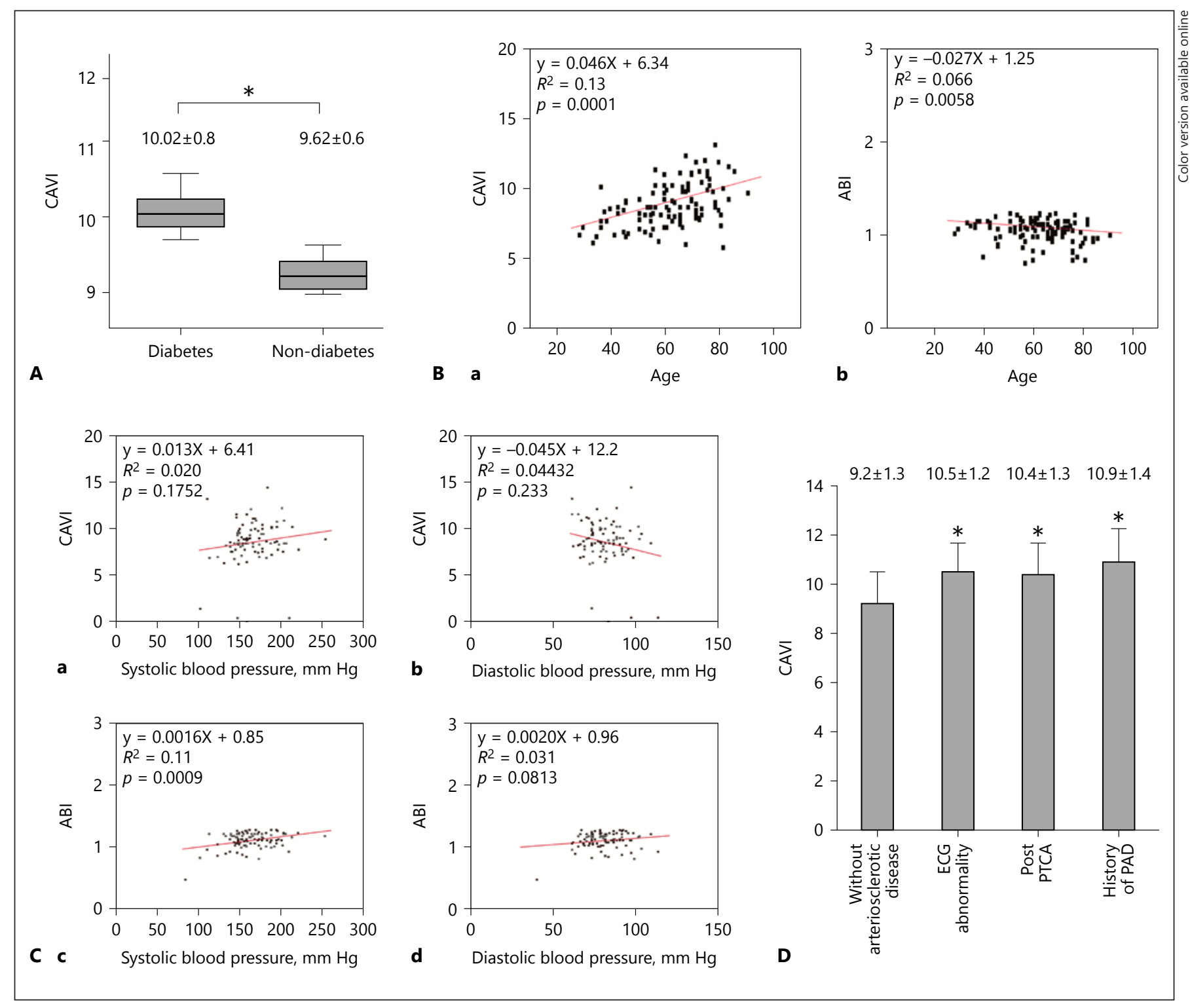

Fig. 1. A Box plots showing the median and dispersion of the CAVI distribution for diabetic patients and others. The lines within boxes represent medians. Lower and upper boundaries of the boxes indicate the 25th and 75th percentiles, and the bars below and above the boxes indicate the 10th and 90th percentiles respectively. ${ }^{*} p<0.05$. B Comparison of age dependency between the CAVI and ABI. In hemodialysis patients $(n=100)$, correlations of age

dialysis. Figure $1 \mathrm{Ba}$ shows a positive relationship between age and the CAVI. The ABI was also significantly correlated with age. Interestingly, the ABI was decreased in older patients (Fig. 1Bb).

Figure $1 \mathrm{C}$ shows the relationship between blood pressure and the CAVI or ABI. With increasing systolic pressure, the CAVI increased slightly (a), and the ABI in- with the CAVI and ABI were studied. C Comparison of blood pressure dependency between the CAVI and ABI. In hemodialysis patients $(n=100)$, correlations of systolic and diastolic pressures with the CAVI and ABI were studied. D CAVI in hemodialysis patients with various arteriosclerotic histories. Data are expressed as means $\pm \operatorname{SD}(n=6-10) . * p<0.05$, when compared with the values for patients without arteriosclerotic disease. 
Table 2. Patients' characteristics and clinical parameters by CAVI quartile

\begin{tabular}{|c|c|c|c|c|c|c|}
\hline & Q1 (6.1-8.2) & Q2 (8.3-9.3) & Q3 (9.4-10.5) & Q4 (10.6-13.4) & $p$ value & \\
\hline Male & $75.0 \pm 44.7$ & $62.5 \pm 50.0$ & $71.4 \pm 46.9$ & $73.3 \pm 45.8$ & 0.879 & ns \\
\hline Age & $57.7 \pm 17.1$ & $54.9 \pm 12.7$ & $62.9 \pm 13.3$ & $70.5 \pm 8.6$ & 0.010 & $*$ \\
\hline Dulation & $18.1 \pm 35.6$ & $11.5 \pm 9.7$ & $28.8 \pm 51.7$ & $27.2 \pm 59.0$ & 0.782 & ns \\
\hline $\mathrm{ABI}$ & $1.13 \pm 0.2$ & $1.08 \pm 0.13$ & $1.08 \pm 0.1$ & $1.08 \pm 0.1$ & 0.706 & ns \\
\hline SPP & $78.3 \pm 21.0$ & $66.0 \pm 22.8$ & $68.7 \pm 14.5$ & $52.9 \pm 10.4$ & 0.037 & $*$ \\
\hline LDL & $96.2 \pm 13.7$ & $96.1 \pm 21.6$ & $93.3 \pm 13.2$ & $93.5 \pm 28.9$ & 0.966 & ns \\
\hline CRP & $0.05 \pm 0.0$ & $0.13 \pm 0.12$ & $0.27 \pm 0.3$ & $0.1 \pm 0.1$ & 0.053 & ns \\
\hline bSBP & $147.8 \pm 13.5$ & $164.1 \pm 23.27$ & $155.3 \pm 16.4$ & $168.8 \pm 26.6$ & 0.179 & ns \\
\hline bDBP & $87.0 \pm 4.3$ & $96.1 \pm 3.6$ & $90.5 \pm 3.9$ & $90.7 \pm 4.2$ & 0.424 & ns \\
\hline aSBP & $162.4 \pm 37.4$ & $179.0 \pm 23.14$ & $177.7 \pm 20.0$ & $165.5 \pm 29.2$ & 0.433 & ns \\
\hline $\mathrm{aDBP}$ & $72.1 \pm 14.3$ & $82.0 \pm 13.0$ & $84.1 \pm 11.1$ & $73.1 \pm 16.4$ & 0.138 & ns \\
\hline
\end{tabular}

Values are expressed as numbers with percentages or the means \pm SD.

$* p<0.05$.

Q, quartile; bSBP, bronchial systolic blood pressure; bDBP, brachial diastolic blood pressure; aSBP, ankle systolic blood pressure; aDBP, ankle diastolic blood pressure; ns, not significant.

The CAVI in hemodialysis patients without a history or signs of arteriosclerotic disease was $9.2 \pm 1.3$ (Fig. 1D). That in arteriosclerotic patients having ischemic change on their electrocardiogram or undergoing percutaneous transluminal coronary angioplasty was significantly higher than the value for those without arteriosclerotic disease. Patients having a history of PAD showed the highest CAVI values. These results suggested that the CAVI could reflect arteriosclerosis of the coronary, femoral, or tibial arteries quantitatively.

Table 2 shows the clinical parameters and the SPP of patients by CAVI quartile. Because the SPP indicates the final pathway of capillary flow through the skin with a laser Doppler probe [13], it has the potential to identify severe limb ischemia in hemodialysis patients [14]. Patients in the highest quartile were older and showed lower SPP. There were no differences in blood pressure among the CAVI quartile groups. These results clearly demonstrated that the CAVI reflects the PAD of hemodialysis patients, independent of their blood pressure values.

\section{Discussion}

CKD, diabetes mellitus, and PAD are associated with a significantly higher risk of death [15], and early detection of PAD in hemodialysis patients is important. The ABI is currently used to evaluate $\mathrm{PAD}$ worldwide. However, high $\mathrm{ABI}$ values are observed in patients with diabetes or renal failure because they may have calcification in lower leg arteries, causing a falsely increased ABI [16]. Indeed, recent reports indicated that false-negative ABI results occur in $17-24 \%$ of limbs of diabetic and hemodialysis patients $[17,18]$.

The Framingham Heart Study showed that about 20\% of symptomatic patients with PAD had diabetes. Conversely, in diabetics, the risk of PAD increased with age, duration of diabetes, and the presence of peripheral neuropathy [19]. Since many patients with diabetes are asymptomatic, the true prevalence of PAD in diabetes is difficult to determine.

Ascertaining the real prevalence of PAD in diabetics may be very beneficial, since the early diagnosis of PAD may facilitate early treatment, and this translates into a better prognosis. Although this is true in the whole diabetic population, this is even more important in the elderly [20]. Moreover, it is likely that patients cared for by vascular surgeons may be different from those cared for by other medical specialists. Unfortunately, current data about the prevalence of PAD in elderly patients with diabetes is very scarce. The ADAN study was performed to assess the prevalence of PAD in an elderly population with diabetes.

The CAVI in hemodialysis patients without arteriosclerotic disease was $9.2 \pm 1.3$. That in arteriosclerotic patients receiving percutaneous transluminal coronary angioplasty or in patients having ischemic change on their ECG was significantly higher than the value for those without coronary arteriosclerotic disease. Patients having diabetes or a history of PAD also had a significantly high-
28

Blood Purif 2019;47(suppl 2):25-30 DOI: $10.1159 / 000496632$
Furuya/Motosugi/Haraguchi/Hanai/Ishii/ Yamaguchi/Kitamura 
er CAVI. These findings suggest that the CAVI might quantitatively reflect the degree of arteriosclerosis in hemodialysis patients.

Previous reports indicated that the reproducibility of the CAVI was high, compared with the general limit for clinical laboratory testing [7]. Measurement is easy and does not need special techniques. Thus, it can be useful for routine studies of hemodialysis patients.

A recent concept of measuring the stiffness of the aorta, femoral artery, and tibial artery as a whole was proposed, and a device for measuring it was also developed. The present study of hemodialysis patients indicated that the CAVI reflects arteriosclerosis. The clinical usefulness of the CAVI will have to be verified by accumulating further clinical data.

\section{Acknowledgment}

We thank the staff of Haraguchi clinic for their efforts in collecting clinical data.

\section{Disclosure Statement}

The authors declare that they have no conflicts of interest to disclose.

\section{Funding Source}

This work was supported by the Japan Society for the Promotion of Science, KAKENHI (15K09424). The authors have nothing to disclose.

\section{Permissions for Image Reproduction}

Not applicable.

\section{Prior Publication}

This is an original work, and has not been published in whole or in part elsewhere and is not under consideration by any other journal.

\section{References}

1 Stehouwer CD, Clement D, Davidson C, Diehm C, Elte JW, Lambert M, Sereni D; EFIM Vascular Medicine Working Group: Peripheral arterial disease: a growing problem for the internist. Eur J Intern Med 2009;20: 132-138.

2 Hackam DG: Medical management of peripheral arterial disease. JAMA 2006;296: 41;author reply 41-42.

3 Brevetti G, Schiano V, Verdoliva S, Silvestro A, Sirico G, De Maio J, Lanero S, Chiariello M: Peripheral arterial disease and cardiovascular risk in Italy. Results of the Peripheral Arteriopathy and Cardiovascular Events (PACE) study. J Cardiovasc Med (Hagerstown) 2006; 7:608-613.

4 Ouriel K: Peripheral arterial disease. Lancet 2001;358:1257-1264.

5 Blacher J, Guerin AP, Pannier B, Marchais SJ, London GM: Arterial calcifications, arterial stiffness, and cardiovascular risk in end-stage renal disease. Hypertension 2001;38:938-942.

6 Lehmann ED: Noninvasive measurements of aortic stiffness: methodological considerations. Pathol Biol (Paris) 1999;47:716-730.

7 Shirai K, Utino J, Otsuka K, Takata M: A novel blood pressure-independent arterial wall stiffness parameter; cardio-ankle vascular index (CAVI). J Atheroscler Thromb 2006;13: 101-107.

8 Takaki A, Ogawa H, Wakeyama T, Iwami T, Kimura M, Hadano Y, Matsuda S, Miyazaki Y, Hiratsuka A, Matsuzaki M: Cardio-ankle vascular index is superior to brachial-ankle pulse wave velocity as an index of arterial stiffness. Hypertens Res 2008;31:1347-1355.

9 Ibata J, Sasaki H, Kakimoto T, Matsuno S, Nakatani M, Kobayashi M, Tatsumi K, Nakano Y, Wakasaki H, Furuta H, Nishi M, Nanjo K: Cardio-ankle vascular index measures arterial wall stiffness independent of blood pressure. Diabetes Res Clin Pract 2008;80:265-270.

10 Yamada T, Ohta T, Ishibashi H, Sugimoto I, Iwata $\mathrm{H}$, Takahashi M, Kawanishi J: Clinical reliability and utility of skin perfusion pressure measurement in ischemic limbs - comparison with other noninvasive diagnostic methods. J Vasc Surg 2008;47:318-323.

11 Hirai T, Sasayama S, Kawasaki T, Yagi S: Stiffness of systemic arteries in patients with myocardial infarction. A noninvasive method to predict severity of coronary atherosclerosis. Circulation 1989;80:78-86.

12 Bramwell JC: Discussion on Hyperpiesis. Proc R Soc Med 1926;19:39-40.

13 Holstein P, Trap-Jensen J, Bagger H, Larsen $\mathrm{B}$ : Skin perfusion pressure measured by isotope washout in legs with arterial occlusive disease. Evaluation of different tracers, comparison to segmental systolic pressure, angiography and transcutaneous oxygen tension and variations during changes in systemic blood pressure. Clin Physiol 1983;3:313-324.

14 Hatakeyama S, Saito M, Ishigaki K, Yamamoto $\mathrm{H}$, Okamoto A, Ishibashi $\mathrm{Y}$, Murasawa $\mathrm{H}$, Imanishi K, Tokui N, Okamoto T, Suzuki Y,
Sugiyama N, Imai A, Kudo S, Yoneyama T, Hashimoto Y, Koie T, Kaminura N, Saitoh H, Funyu T, Ohyama C: Skin perfusion pressure is a prognostic factor in hemodialysis patients. Int J Nephrol 2012:385274.

15 Liew YP, Bartholomew JR, Demirjian S, Michaels J, Schreiber MJ Jr: Combined effect of chronic kidney disease and peripheral arterial disease on all-cause mortality in a high-risk population. Clin J Am Soc Nephrol 2008;3: 1084-1089.

16 Hirsch AT, Haskal ZJ, Hertzer NR, Bakal CW, Creager MA, Halperin JL, Hiratzka LF, Murphy WR, Olin JW, Puschett JB, Rosenfield KA, Sacks D, Stanley JC, Taylor LM Jr, White CJ, White J, White RA, Antman EM, Smith SC Jr, Adams CD, Anderson JL, Faxon DP, Fuster V, Gibbons RJ, Hunt SA, Jacobs AK, Nishimura R, Ornato JP, Page RL, Riegel B; American Association for Vascular Surgery; Society for Vascular Surgery; Society for Cardiovascular Angiography and Interventions; Society for Vascular Medicine and Biology; Society of Interventional Radiology; ACC/AHA Task Force on Practice Guidelines Writing Committee to Develop Guidelines for the Management of Patients With Peripheral Arterial Disease; American Association of Cardiovascular and Pulmonary Rehabilitation; National Heart, Lung, and Blood Institute; Society for Vascular Nursing; TransAtlantic Inter-Society Consensus; Vascular Disease Foundation: ACC/AHA 
2005 Practice Guidelines for the management of patients with peripheral arterial disease (lower extremity, renal, mesenteric, and abdominal aortic): a collaborative report from the American Association for Vascular Surgery/Society for Vascular Surgery, Society for Cardiovascular Angiography and Interventions, Society for Vascular Medicine and Biology, Society of Interventional Radiology, and the ACC/AHA Task Force on Practice Guidelines (Writing Committee to Develop Guidelines for the Management of Patients With Peripheral Arterial Disease): endorsed by the American Association of Cardiovascular and Pulmonary Rehabilitation; National Heart, Lung, and Blood Institute; Society for Vascular Nursing; TransAtlantic Inter-Society Consensus; and Vascular Disease Foundation. Circulation 2006; 113:e463-654.

17 Carter SA: Ankle and toe systolic pressures comparison of value and limitations in arterial occlusive disease. Int Angiol 1992;11: 289-297.

18 Larsson J, Apelqvist J, Castenfors J, Agardh $\mathrm{CD}$, Stenstrom A: Distal blood pressure as a predictor for the level of amputation in diabetic patients with foot ulcer. Foot Ankle 1993;14:247-253.

19 Murabito JM, D'Agostino RB, Silbershatz H, Wilson WF: Intermittent claudication. A risk profile from The Framingham Heart Study. Circulation 1997;96:44-49.

20 Escobar C, Blanes I, Ruiz A, Vinuesa D, Montero M, Rodriguez M, Barbera G, Manzano L: Prevalence and clinical profile and management of peripheral arterial disease in elderly patients with diabetes. Eur J Intern Med 2011; 22:275-281. 